\title{
Evidence for weak magnetic fields in early-type emission stars $^{\star}$
}

\author{
S. Hubrig ${ }^{1, \star \star}$, R.V. Yudin ${ }^{2,3}$, M. Pogodin ${ }^{2,3}$, M. Schöller ${ }^{1}$, and G.J. Peters ${ }^{4}$ \\ 1 ESO, Casilla 19001, Santiago, Chile \\ 2 Pulkovo Observatory, Saint-Petersburg, 196140, Russia \\ 3 Isaac Newton Institute of Chile, Saint-Petersburg Branch, Russia \\ ${ }^{4}$ Space Sciences Center, University of Southern California, University Park, Los Angeles, CA 90089-1341, USA
}

Received 2007, accepted 2007

Published online later

Key words stars: emission-line, Be - stars: magnetic fields - stars: circumstellar matter - techniques: polarimetric

We report the results of our study of magnetic fields in a sample of 15 Be stars using spectropolarimetric data obtained at the European Southern Observatory with the multi-mode instrument FORS 1 installed at the $8 \mathrm{~m}$ Kueyen telescope. We detect weak photospheric magnetic fields in four stars, HD 56014, HD 148184, HD 155806, and HD 181615. We note that for HD 181615 the evolutionary status is not obvious due to the fact that it is a binary system currently observed in the initial rapid phase of mass exchange between the two components. Further, we notify the possible presence of distinct circular polarisation features in the circumstellar components of Ca II H\&K in three stars, HD 58011, HD 117357, and HD 181615, hinting at a probable presence of magnetic fields in the circumstellar mass loss disks of these stars. We emphasize the need for future spectropolarimetric observations of Be stars with detected magnetic fields to study the temporal evolution of their magnetic fields and the correlation of magnetic field properties with dynamical phenomena taking place in the gaseous circumstellar disks of these stars.

(C) 2006 WILEY-VCH Verlag GmbH \& Co. KGaA, Weinheim

\section{Introduction}

Be stars are defined as rapidly rotating main sequence stars showing normal B-type spectra with superposed Balmer emissions. Further, these stars are characterized by episodic dissipation and formation of a new circumstellar (CS) disk-like environment, non-radial pulsations and photometric and spectroscopic variability. A number of physical processes in classical Be stars (e.g., angular momentum transfer to a CS disk, channeling stellar wind matter, accumulation of material in an equatorial disk, etc.) are more easily explainable if magnetic fields are invoked. In recent years this conclusion was strengthened by numerous theoretical works (e.g., Cassinelli et al. 2002, Maheswaran 2003, Brown et al. 2004). A detailed discussion concerning the mechanisms involved in formation and evolution of disks around $\mathrm{Be}$ stars can be found in a recent publication by Owocki (2006).

To date we know only three Be stars with magnetic field detections: $\omega$ Ori $(80 \pm 40 \mathrm{G}-$ Neiner et al. 2003), $\beta$ Cep $(95 \pm 8 \mathrm{G}-$ Donati et al. 2001), and $16 \mathrm{Peg}(-156 \pm 31 \mathrm{G}$ - Hubrig et al. 2006). These results are cited in the literature as observational evidence for the existence of magnetic fields in Be stars (e.g. Brown et al. 2004). However, $\beta$ Cep has been shown to be a binary star, where the $\mathrm{H} \alpha$ emission line and the magnetic field originate in two different components (e.g. Schnerr et al. 2006), and the magnetic field

\footnotetext{
* Based on observations collected at ESO, Paranal, Chile (ESO programmes Nos. 075.D-0507 and 077.D-0406).

$\star \star$ Corresponding author: e-mail: shubrig@eso.org
}

measured in $\omega$ Ori is below a $3 \sigma$ threshold. A longitudinal magnetic field at a level larger than $3 \sigma$ has been diagnosed for the Be star 16 Peg (Hubrig et al. 2006). This star has $v \sin i=104 \mathrm{~km} / \mathrm{s}$ and was classified as a Be star by Merrill \& Burwell (1943) due to the detection of double emission in $\mathrm{H} \alpha$. However, the emission was not confirmed by subsequent observations, and so the question of the presence of magnetic fields in classical Be stars remains open.

It is quite possible that the difficulty of detecting magnetic fields in Be stars is quite similar to that discussed for young Herbig Ae/Be stars with accretion disks by Hubrig et al. (2007). However, in contrast to Herbig stars showing evidence of magnetically mediated disk accretion, the Be disks originate from ejection or decretion of mass from the rapidly rotating B stars. The observed spectral lines may form over a relatively large volume, and the magnetic field topology is likely rather complex. The presence of a mixture of photospheric and CS magnetic fields could drive the net line-of-sight magnetic flux to near zero values. Also, even if there are very strong, small scale magnetic fields distributed over the surface of the star, these could go undetected in our measurements, as the measured mean longitudinal magnetic field $\left\langle B_{z}\right\rangle$ is the average over the stellar hemisphere visible at the time of observation of the component of the magnetic field parallel to the line of sight, weighted by the local emergent spectral line intensity.

Below, we report our results of the study of magnetic fields in a sample of $15 \mathrm{Be}$ stars carried out with the multimode instrument FORS 1 installed at the $8 \mathrm{~m}$ Kueyen telescope at the VLT. 
Table 1 The sample of stars observed and the resulting measurements of magnetic fields.

\begin{tabular}{rccccccccc}
\hline \hline HD & $\begin{array}{c}\text { Other } \\
\text { identifier }\end{array}$ & $\mathrm{V}$ & MJD & Sp. Type & $\begin{array}{c}v \sin i \\
{[\mathrm{~km} / \mathrm{s}]}\end{array}$ & $\begin{array}{c}T_{\text {eff }} \\
{[\mathrm{K}]}\end{array}$ & $\begin{array}{l}\log g \\
\end{array}$ & $\begin{array}{c}\left\langle B_{z}\right\rangle \\
{[\mathrm{G}]}\end{array}$ & $\begin{array}{c}\text { Circumstellar } \\
\text { Zeeman features }\end{array}$ \\
\hline 53367 & V750 Mon & 7.0 & 53503.496 & B0IVe & 86 & 28000 & 3.9 & $-9 \pm 30$ & - \\
56014 & EW CMa & 4.7 & 53512.469 & B3IIIp+sh & 144 & 20000 & 3.8 & $-\mathbf{1 4 6} \pm \mathbf{3 2}$ & - \\
56139 & $\omega$ CMa & 4.0 & 53502.547 & B2.5Ve & 109 & 22000 & 4.0 & $-31 \pm 24$ & - \\
57219 & NW Pup & 5.1 & 53630.820 & B2Vne & 117 & 23000 & 4.0 & $+80 \pm 32$ & - \\
58011 & NN CMa & 7.2 & 53502.529 & B1Vnep & 18 & 25000 & 3.9 & $+135 \pm 48$ & H+K \\
58050 & OT Gem & 6.4 & 53629.914 & B2Ve & 123 & 23000 & 4.0 & $-101 \pm 35$ & - \\
79351 & a Car & 3.4 & 53454.590 & B2.5V & 35 & 22000 & 4.0 & $-7 \pm 59$ & - \\
105435 & $\delta$ Cen & 2.6 & 53474.622 & B2IVne & 207 & 23000 & 3.9 & $-30 \pm 30$ & - \\
117357 & CD-61 3819 & 9.1 & 53508.491 & B0.5IIIne & 78 & 27000 & 3.9 & $+7 \pm 59$ & H+K \\
137432 & HR 5636 & 5.4 & 53531.660 & B4Ve & 127 & 18000 & 4.1 & $-58 \pm 24$ & - \\
148184 & $\chi$ Oph & 4.4 & 53531.722 & B1.5Vpe & 139 & 24000 & 4.0 & $+\mathbf{8 3} \pm \mathbf{2 1}$ & - \\
148184 & $\chi$ Oph & 4.4 & 53862.380 & B1.5Vpe & 139 & 24000 & 4.0 & $\mathbf{+ 1 3 6} \pm \mathbf{1 6}$ & - \\
148259 & OZ Nor & 7.4 & 53571.601 & B2Ve & 86 & 23000 & 4.0 & $+116 \pm 49$ & - \\
153261 & V828 Ara & 6.3 & 53531.750 & B2IVe & 184 & 23000 & 3.9 & $-33 \pm 41$ & - \\
155806 & V1075 Sco & 5.6 & 53531.775 & O7.5 IIIe & 116 & 34000 & 3.8 & $-\mathbf{1 1 5} \pm \mathbf{3 7}$ & - \\
181615 & $v$ Sgr & 4.6 & 53519.910 & B2Vpe & 69 & 23000 & 4.0 & $\mathbf{+ 3 8} \pm \mathbf{1 0}$ & H+K \\
\hline
\end{tabular}

\section{Analysis}

The observations have been carried out in April-September 2005 in service mode. Using the narrowest available slit width of $0 ! .4$ the achieved spectral resolving power of the FORS 1 spectra obtained with the GRISM $1200 \mathrm{~g}$ was about 4000 in the spectral region $\lambda \lambda 3850-5000 \AA$. Each observation consisted of 8-10 subexposures with a typical duration of the order of tens of seconds for each subexposure. One additional observation of the Be star HD 148194 ( $\chi$ Oph) was obtained in May 2006 with the same instrument and GRISM 600B using the narrowest available slit width of 0.4 to obtain a resolving power of about 2000.

A detailed description of the assessment of the longitudinal magnetic field measurements using FORS 1 is presented in our previous papers (e.g., Hubrig et al. 2004a, $2004 b$, and 2004c, and references therein). The errors of the measurements of the polarization have been determined from photon counting statistics and have been converted to errors of field measurements.

\section{Results}

The results of our analysis are summarised in Table 1 In the first four columns we give the HD number of the target, another identifier, the V magnitude, and the modified Julian date of the observations. In the next two columns follow the spectral type and the $v \sin i$ value, which were both taken from Yudin (2001). In columns seven and eight we present $T_{\text {eff }}$ and $\log g$ values used to calculate the synthetic spectra, and in column nine we list the measured mean longitudinal magnetic fields $\left\langle B_{z}\right\rangle$. The mean longitudinal magnetic field is diagnosed from the slope of a linear regression of $V / I$ versus the quantity $-\frac{g_{\text {eff }} e}{4 \pi m_{e} c^{2}} \lambda^{2} \frac{1}{I} \frac{\mathrm{d} I}{\mathrm{~d} \lambda}\left\langle B_{z}\right\rangle+V_{0} / I_{0}$. We show an example of the regression detection for the magnetic field in HD 56014 in Fig. 1] Our experience from studying a large

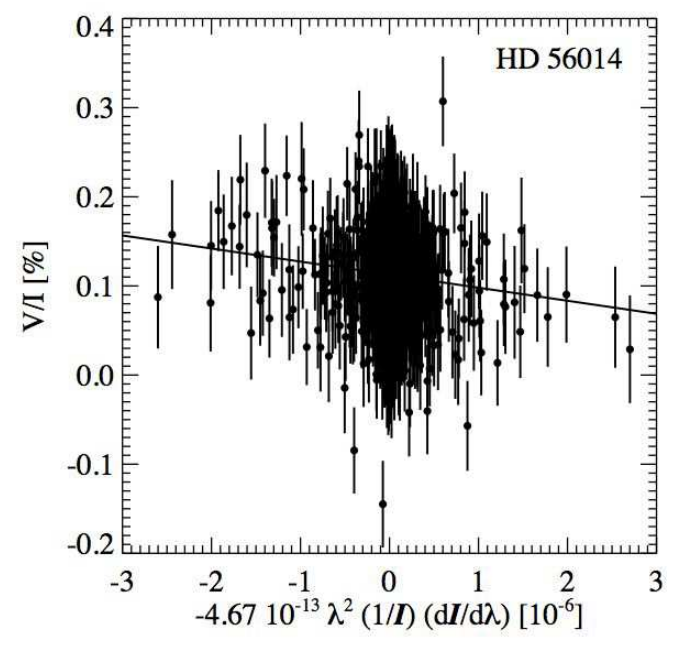

Fig. 1 Regression detection of the magnetic field in HD 56014.

sample of magnetic and non-magnetic Ap and Bp stars revealed that this regression technique is very robust and that detections with $\left\langle B_{z}\right\rangle>3 \sigma_{z}$ result only for stars possessing magnetic fields. A longitudinal magnetic field at a level larger than $3 \sigma$ has been detected in four stars, HD 56014, HD 148184, HD 155806, and HD 181615 (also indicated in bold face in Table 1). Also, an inspection of the Stokes V spectra of these four stars reveals noticeable Zeeman features at the position of numerous spectral lines. As an example, we present in Fig. 2 the Stokes I and V spectra for HD 148184 and HD 155806 in the spectral region around the line He I $\lambda 4471.5 \AA$.

According to Porter \& Rivinius (2003), the photospheric spectra of Be stars are frequently superposed by strong absorption spectra from Be CS disks. For three stars in our 


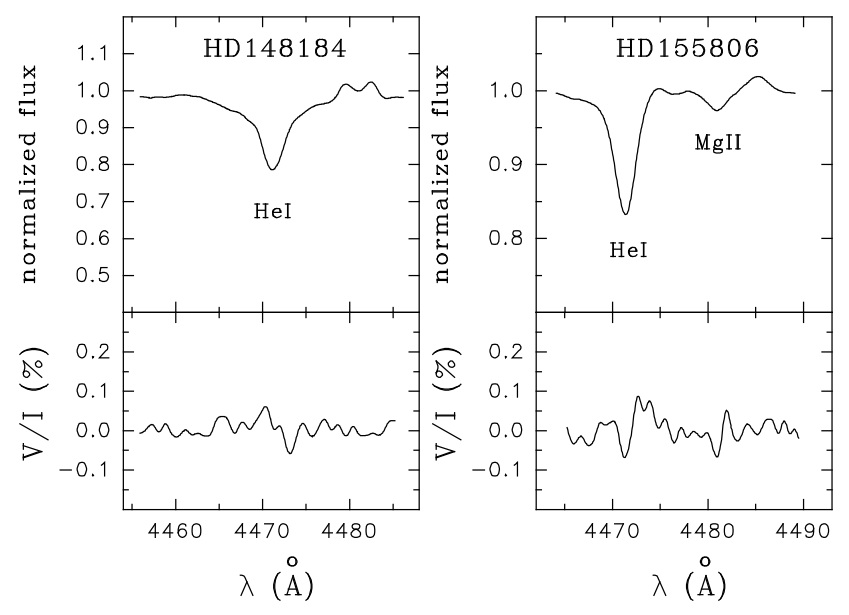

Fig. 2 Stokes I and V spectra of HD 148184 and HD 155806 in the spectral region around the line $\mathrm{He} \mathrm{I} \lambda$ $4471.5 \AA$.

sample, HD 58011, HD 117357, and HD 181615, we noticed the presence of distinctive circular polarization signatures detected in the Stokes V spectra of the Ca II H\&K lines which appear unresolved at the low spectral resolution achiev able with FORS 1 (R 4000), denoted in column 10 of Table 1 . The profiles of these Ca lines in the FORS 1 spectra taken in integral light are deeper than predicted by synthetic spectra computed with the code SYNTH + ROTATE developed by Piskunov (1992). In Fig. 3 we present Stokes I spectra (upper panel) and Stokes V spectra (lower panel) around the Ca II resonance doublet for the stars HD 58011, HD 117357, and HD 181615. HD 58011, the Be star with numerous strong emission lines in the visible spectrum, was reported to be variable with an amplitude of 0.25 after the Hipparcos mission by Adelman, Mayer \& Rosidivito (2000). However, the magnetic field is determined only at a $2.8 \sigma$ level $\left(\left\langle B_{z}\right\rangle=+135 \pm 48 \mathrm{G}\right)$. Not much is known about the rather faint Be star HD $117357\left(\mathrm{~m}_{\mathrm{V}}=9.1\right)$. Wiegert \& Garrison (1998) report about the presence of variable emission in Balmer hydrogen lines. In our Stokes I spectra the emission lines are quite weak, and the magnetic field is not detectable. A detailed discussion on the peculiar spectrum of the system HD 181615 was presented by Koubský et al. (2006) who suggested that the visible spectrum may actually be a combined spectrum of the disk rim and disk face. As our FORS 1 spectra are taken at a rather low resolving power, it is presently not possible to correctly ascertain the origin of these Ca II H\&K lines. Additional high-resolution high signal-to-noise spectroscopic observations are needed to study the $\mathrm{Ca}$ line profiles to be able to decide whether they are formed in the CS disks around these two stars.

Below we present brief notes on individual objects with detected photospheric magnetic fields.

\subsection{HD 56014}

Rivinius, Štefl \& Baade (2006) describe this star as a conventional Be shell star with narrow absorption lines and with central quasi emission bumps in photospheric lines detected in several Fe II lines and in Mg II $\lambda 4481 \AA$. The magnetic field for this star is detected at a $4.5 \sigma$ level $\left(\left\langle B_{z}\right\rangle=\right.$ $-146 \pm 32 \mathrm{G})$. According to the Washington Double Star Catalog (WDS - Worley \& Douglass 1997) and Mason et al. (1997) this object is a visual binary (WDS 07143-2621) with an angular separation of 0.150 and an orbital period of about $180 \mathrm{yr}$.

\subsection{HD 148184}

This Be star with numerous strong emission lines in the Stokes I spectrum belongs to Upper Scorpius, which is the youngest of the three subgroups that form the ScorpiusCentaurus association. The variability of the $\mathrm{H} \alpha$ line profiles has been reported by Austin et al. (2004). Hubert \& Floquet (1998) discovered cyclic behaviour of the Hipparcos photometry, but could only constrain the period to be $>0.45 \mathrm{~d}$. The magnetic field for this star is detected at a $4 \sigma$ level $\left(\left\langle B_{z}\right\rangle=+83 \pm 21 \mathrm{G}\right)$ and at a $8 \sigma$ level $\left(\left\langle B_{z}\right\rangle=+136 \pm 16 \mathrm{G}\right)$.

\subsection{HD 155806}

This is the hottest star in our sample with spectral type O7.5IIIe and is therefore the earliest known star showing emission lines typically seen in Be stars (e.g. Negueruela, Steele \& Bernabeu 2004). The magnetic field for this star is detected at a level of $3.1 \sigma\left(\left\langle B_{z}\right\rangle=-115 \pm 37 \mathrm{G}\right)$.

\subsection{HD 181615}

This system is a very rare emission-line binary system of a strange spectral type and complexity. The existence of strong $\mathrm{H} \alpha$ emission in the visible spectrum was reported by Campbell (1895) and other investigators. Very recently, Koubský et al. (2006) concluded that this system with an orbital period of $138 \mathrm{~d}$ is one of very few known binary systems observed in the initial rapid phase of mass exchange between the two components. From the photometric and spectroscopic observations Koubský et al. infer the presence of bipolar jets perpendicular to the orbital plane, similar to those found for $\beta \mathrm{Lyr}$, and argue that the peculiar character of the line spectrum of the brighter component could also be understood as originating from a pseudo-photosphere of an optically thick disk. The magnetic field is detected at a level of $3.8 \sigma\left(\left\langle B_{z}\right\rangle=+38 \pm 10 \mathrm{G}\right)$.

\section{Discussion}

The detected magnetic fields in the studied Be stars are rather weak, with the largest longitudinal magnetic field of $-146 \mathrm{G}$ measured in the Be star HD 56014. These results suggest that strong large scale organized magnetic fields are not common among the group of Be type stars. Further, we noticed the possible presence of distinct circular polarisation features in the CS components of Ca II H\&K in two Be stars, 

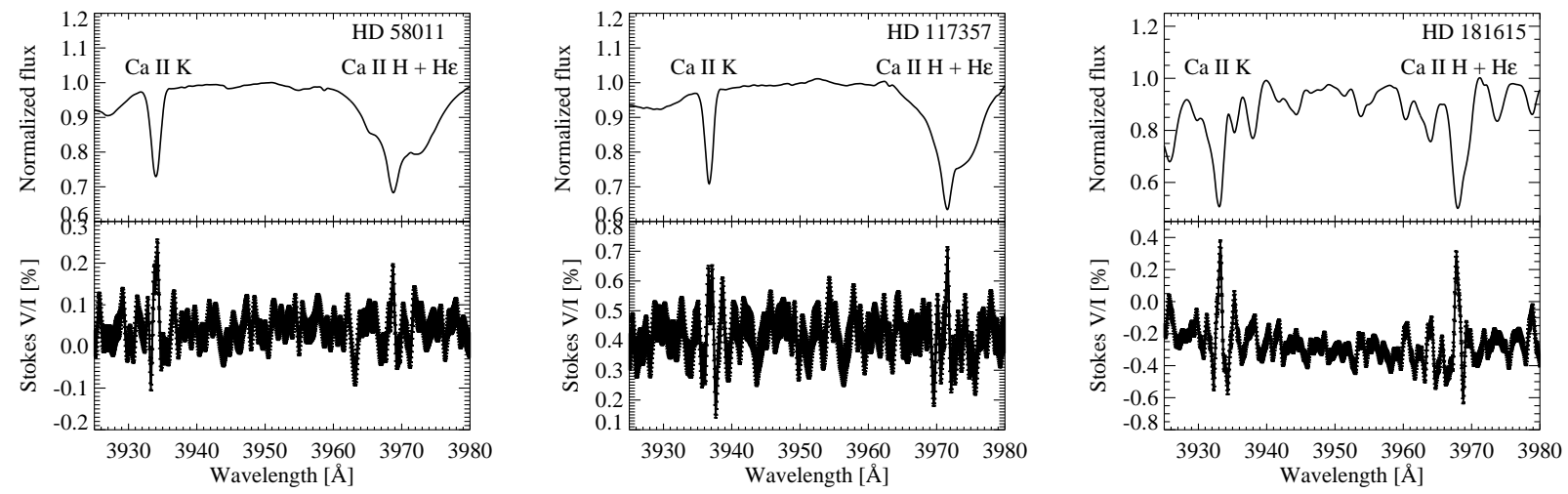

Fig. 3 Stokes I and V spectra of HD 58011, HD 117357, and HD 181615 around the Ca II doublet. The thickness of the plotted lines in the Stokes V spectra corresponds to the uncertainty of the measurement of polarization determined from photon noise.

HD 58011 and HD 117357, as well as in HD 181615, indicating the possible presence of magnetic fields in the CS mass loss disks. Interestingly, similar type CS components in Ca II H\&K lines have recently been discovered by us from FORS 1 observations of Herbig Ae/Be stars (Hubrig et al. 2007). The CS Ca II H\&K line profiles in Stokes I spectra of Herbig Ae/Be stars are frequently quite complex and consist of several components which are usually assumed to be formed at the base of the stellar wind and in the accretion gaseous flow. A future careful high resolution high signal-to-noise spectropolarimetric study of the temporal behaviour of the Zeeman features in the Stokes V spectra will allow to get the highly desirable insight into the nature of gaseous disks of these two Be stars.

During our observations the exposure times for each target were rather short, of the order of 15-20 min. Thus, not much can be inferred with respect to the variability and evolution of their magnetic fields and the correlation of magnetic field properties with dynamical phenomena taking place in these stars. To constrain the structure of magnetic fields in Be stars and to probe the presence of localized transient magnetic fields suggested by several previous studies (e.g., Mathys \& Smith 2000 and references therein), the magnetic field measurements should be carried out over short (minutes) and long (rotational periods) time scales. The existence of small scale, i.e. highly non-dipole magnetic fields has been suggested by X-ray observations of flaring events (e.g. Smith, Robinson \& Corbet 1998).

\section{Acknowledgments}

MP was supported partly by the RSSI grant 07-02-00535.

\section{References}

Adelman, S.J., Mayer, M.R., Rosidivito, M.A.: 2000, IBVS 5008, 1
Austin, S.J., Dunlap, B., Franklin, M., Hoggard, T., Hoskins, J.S.: 2004, AAS 36, 1426

Brown, J.C., Telfer, D., Li, Q., Hanuschik, R., Cassinelli, J.P., Kholtygin, A.: 2004, MNRAS 352, 1061

Campbell, W.W.: 1895, ApJ 2, 177

Cassinelli, J.P., Brown, J.C., Maheswaran, M., Miller, N.A., Telfer, D.C.: 2002, ApJ 578, 951

Donati, J.-F., Wade, G.A., Babel, J., Henrichs, H.f., de Jong, J.A., Harries, T.J.: 2001, MNRAS 326, 1265

Hubert, A.M., Floquet, M.: 1998, A\&A 335, 565

Hubrig, S., Briquet, M., Schöller, M., De Cat, P., Mathys, G., Aerts, C.: 2006, MNRAS 369, L61

Hubrig, S., Kurtz, D.W., Bagnulo, S., Szeifert, T., Schöller, M., Mathys, G., Dziembowski, W.A.: 2004a, A\&A 415, 661

Hubrig, S., Pogodin, M.A., Yudin, R.V., Schöller, M., Schnerr, R.S.: 2007, A\&A 463, 1039

Hubrig, S., Szeifert, T., Schöller, M., Mathys, G., Kurtz, D.W.: 2004b, A\&A 415, 685

Hubrig, S., Schöller, M., Yudin, R.V.: 2004c, A\&A 428, 1

Koubský, P., Harmanec, P., Yang, S., Netolický, M., Škoda, P., Šlechta, M., Korčáková, D.: 2006, A\&A 459, 849

Maheswaran, M:, 2003, ApJ 592, 1156

Mason, B.D., Ten Brummelaar, T., Gies, D.R., Hartkopf, W.I., Thaller, M.L.: 1997, AJ 114, 2112

Mathys, G., Smith, M.A.: 2000, ASPC 214, 316 In: “The Be Phenomenon in Early-Type Stars", IAU Colloquium 175, ASP Conference Proceedings, Vol. 214, edited by Myron A. Smith and Huib F. Henrichs. Astronomical Society of the Pacific, ISBN 1-58381-045-5, 2000, p. 316

Merrill, P.W., Burwell, C.G.: 1943, ApJ 98, 153

Negueruela, I., Steele, I.A., Bernabeu, G.: 2004, AN 325, 749

Neiner, C., Hubert, A.-M., Frémat, Y., Floquet, M., Jankov, S., Preuss, O., Henrichs, H.F., Zorec, J.: 2003, A\&A 409, 275

Owocki, S.: 2006, In: "Stars with the B[e] Phenomenon". ASP Conference Series, Vol. 355, Proceedings of the Conference Held 10-16 July, 2005, at the Isle of Vlieland, The Netherlands. San Francisco: Astronomical Society of the Pacific, 2006, p. 219

Piskunov, N.E.: 1992, In: "Stellar Magnetism”, Proceedings of international meeting on the problem "Physics and evolution of stars", held in Nizhnij Arkhyz 30 September - 5 October 1991. Edited by Yu.V. Glagolevskij and I.I. Romanyuk. Sankt Petersburg: "NAUKA", Sankt-Petersburg branch, 1992, p. 92 
Porter, J.M., Rivinius, T.: 2003, PASP 115, 1153

Rivinius, T., Štefl, S., Baade, D.: 2006, A\&A 459, 137

Schnerr, R.S., Henrichs, H.F., Oudmaijer, R.D., Telting, J.H.: 2006, A\&A 459, L21

Smith, M.A., Robinson, R.D., Corbet, R.H.D.: 1998, ApJ 503, 877

Wiegert, P., Garrison, R.F.: 1998, JRASC 92, 134

Worley, C.E., Douglass, G.G.: 1997, A\&AS 125, 523

Yudin, R.V.: 2001, A\&A 368, 912 